\title{
Evaluating the impact of chemotherapy-induced peripheral neuropathy symptoms (CIPN-sx) on perceived ability to work in breast cancer survivors during the first year post-treatment
}

\author{
Noah R. Zanville ${ }^{1,2} \cdot$ Kelly N. H. Nudelman ${ }^{2,3,4}$ • Dori J. Smith ${ }^{4}$ Diane Von Ah $^{1}$ • \\ Brenna C. McDonald ${ }^{4,5,6}$ • Victoria L. Champion ${ }^{1,2,5}$ • Andrew J. Saykin ${ }^{3,4,5,6}$
}

Received: 4 February 2016 / Accepted: 3 July 2016 /Published online: 28 July 2016

(C) The Author(s) 2016. This article is published with open access at Springerlink.com

\begin{abstract}
Purpose To describe the impact of chemotherapy-induced peripheral neuropathy symptoms (CIPN-sx) on breast cancer survivors' (BCS) perceived ability to work post-treatment. Methods The sample included 22 chemotherapy-treated $(\mathrm{Ctx}+)$ and 22 chemotherapy-naïve $\left(\mathrm{Ctx}^{-}\right)$female BCS. Data was collected at the following three time points: baseline (post-surgery, pre-chemotherapy), 1 month $(1 \mathrm{M})$ post-chemotherapy, and approximately 1 year (1 Y) later. The presence, frequency, number, and severity of CIPN-sx were selfreported using the Functional Assessment of Cancer Therapy/Gynecologic Oncology Group-Neurotoxicity questionnaire (FACT/GOG-Ntx) version 4, a validated 11-item CIPN measure. Perceived ability to work was measured using an item from the Functional Well-Being subscale of the FACT/GOG-Ntx.
\end{abstract}

Noah R. Zanville

nrzanvil@umail.iu.edu

1 Department of Community \& Health Systems, School of Nursing, Indiana University, Indianapolis, IN, USA

2 Training in Research for Behavioral Oncology and Cancer Control Program, Indiana University School of Nursing, Indianapolis, IN, USA

3 Department of Medical and Molecular Genetics, School of Medicine, Indiana University, Indianapolis, IN, USA

4 Department of Radiology and Imaging Sciences, Indiana University School of Medicine, Indianapolis, IN, USA

5 Indiana University Melvin and Bren Simon Cancer Center, Indiana University, School of Medicine, Indianapolis, IN, USA

6 Indiana Alzheimer Disease Center, Indiana University School of Medicine, Indianapolis, IN, USA
Results At 1 Y, more than $50 \%$ of $\mathrm{Ctx}+$ reported discomfort, numbness, or tingling in their hands or feet; weakness; or difficulty feeling small objects. The presence, number, and severity of these symptoms were correlated with being less able to work for $\mathrm{Ctx}+$ at $1 \mathrm{M}$ but not 1 Y. Results of a regression analysis using CIPN-sx to predict work ability found that models combining (1) hand numbness and trouble feeling small objects, (2) trouble buttoning buttons and trouble feeling small objects, (3) foot numbness and foot pain, (4) foot numbness and trouble walking, and (5) trouble hearing and hand pain each predicted survivors who were "not at all" able to work at $1 \mathrm{M}$.

Conclusions Unresolved CIPN-sx may play a role in challenges working for BCS post-treatment. These findings highlight the need for research to explore the impact that CIPN-sx have on BCS' ability to work, as well as the development of interventions to improve work function in BCS with CIPN-sx.

Keywords Breast cancer $\cdot$ Chemotherapy $\cdot$ Peripheral neuropathy · CIPN · Survivorship · Symptoms · Work · Perceived occupational function

$\begin{array}{ll}\text { Abbreviations } \\ 1 \mathrm{M} & \begin{array}{l}\text { One-month time point, approximately } \\ 1 \text { month post-chemotherapy treatment } \\ \text { completion } \\ \text { One year time point, approximately } 1 \\ \text { year after 1-M time point }\end{array} \\ \text { BL } & \begin{array}{l}\text { Baseline time point, post-surgery, } \\ \text { pre-chemotherapy } \\ \text { Chemotherapy-induced peripheral } \\ \text { neuropathy }\end{array} \\ \text { CIPN } & \end{array}$

Abbreviations 


$\begin{array}{ll}\text { CIPN-sx } & \begin{array}{l}\text { Chemotherapy-induced peripheral } \\ \text { neuropathy symptoms }\end{array} \\ \mathrm{Ctx}+ & \begin{array}{l}\text { Breast cancer patients who received } \\ \text { chemotherapy during treatment }\end{array} \\ \mathrm{Ctx}- & \begin{array}{l}\text { Breast cancer patients who did not receive } \\ \text { chemotherapy during treatment }\end{array} \\ \mathrm{FACT} / \mathrm{GOG}- & \begin{array}{l}\text { Functional Assessment of Cancer Therapy/ } \\ \text { Gynecologic Oncology Group-Neurotoxicity } \\ \text { Ntx }\end{array} \\ & \text { questionnaire }\end{array}$

\section{Introduction}

For the majority of adults in the U.S., work is an essential part of life. Breast cancer survivors (BCS) are no exception; an estimated $60-90 \%$ of BCS return to work full-or part-time after completing cancer treatment $[1,2]$, and a growing number of BCS work throughout their cancer treatment as well [3, 4]. Beyond the simple economic necessity of working, for many survivors, work offers a vital source of self-esteem, structure, and social support [5]. Unfortunately, for many BCS, side effects related to their cancer treatments can interfere with their ability to return to work and regain pretreatment levels of occupational function [6-8].

One side effect of cancer treatment that has received comparatively little attention in the discussion of work among BCS is chemotherapy-induced peripheral neuropathy (CIPN), a form of nerve damage associated with a number of commonly used cancer therapies. Current estimates suggest that $30 \%$ or more of BCS receiving chemotherapy develop some degree of CIPN during treatment depending on the agents, regimen, and individual risk factors [9, 10]. While research has begun to illustrate the potentially serious impact that CIPN symptoms (CIPN-sx) can have on women's quality of life [11-13], ability to complete treatment [14, 15], and ability to perform daily activities $[16,17]$, the impact of CIPN-sx on survivors' ability to work is only beginning to be investigated.

This is troubling for several reasons. First, women make up nearly half of the U.S. labor force [18] and more than $99 \%$ of cases of breast cancer in the U.S. [19]. In many of these cases, use of neurotoxic chemotherapy is standard, putting more and more BCS at risk for developing CIPN. Second, studies have shown that the majority of BCS resume work after treatment [20] but often with significant difficulty [21]. Issues ranging from fatigue, physical limitations, and cognitive symptoms have all been implicated in this difficulty returning to pretreatment levels of occupational functioning [5, 20], but the role that CIPN-sx may play in this difficulty is unclear. This lack of clarity is particularly troubling because $23-86 \%$ of BCS who develop CIPN-sx during treatment continue to report CIPN-sx after treatment [22-27], when return to work is likely. Thirdly, while a number of studies have shown that the severity of CIPN-sx is associated with disruptions in treatment $[14,15]$, poorer quality of life [28], and greater use of health care resources [29], it is not clear whether the presence, frequency, severity, or total number of CIPN-sx is the best predictor of difficulty working post-treatment.

The purpose of this analysis was to evaluate the impact that the presence, frequency, number, and severity of CIPN-sx have on BCS' perceived ability to work post-treatment. Specific aims were to (1) compare the presence, frequency, number, and severity of CIPN-sx in BCS exposed to chemotherapy $(\mathrm{Ctx}+)$ to $\mathrm{BCS}$ whose treatment did not include chemotherapy $\left(\mathrm{Ctx}^{-}\right)$; (2) compare perceived ability to work between $\mathrm{Ctx}+$ and $\mathrm{Ctx}-$ and determine whether the presence, severity, and total number of CIPN-sx survivors reported were associated with their ability to work during the first year posttreatment; and (3) explore which combinations of CIPN-sx best predict survivors' ability to work post-treatment.

\section{Methods}

\section{Sample and eligibility}

Data for the analysis came from a recent longitudinal study evaluating the effect of cancer and cancer treatment on cognitive function in $\mathrm{Ctx}+$ and $\mathrm{Ctx}^{-}$women with non-metastatic breast cancer (i.e., stages 0 -IIIc). The original study also included demographically matched healthy controls, as previously described [30-32]. Healthy controls were not evaluated for CIPNsx and, therefore, were not included in the analysis.

Participants were recruited from the Indiana University Melvin and Bren Simon Cancer Center's recruitment core and affiliated clinical sites. Approval for the study was granted by the Indiana University Institutional Review Board. Written informed consent was collected from all participants. Data was collected at the following three time points: (1) baseline (after breast surgery but before radiation, chemotherapy, or anti-estrogen treatment), (2) approximately 1 month (1 M) after completing chemotherapy, and (3) approximately 1 year (1 Y) after the $1 \mathrm{M}$ visit. Approximately one third of Ctx+ received neo-adjuvant chemotherapy and were surgery and treatment naïve at baseline.

The sample for this analysis consisted of $22 \mathrm{Ctx}^{-}$and 22 $\mathrm{Ctx}+$ women with non-metastatic breast cancer, ages 69 or younger. Exclusion criteria included a self-reported history of prior cancer; substance abuse; and other medical, neurological, and psychiatric risk factors with the potential to affect central or peripheral neurological structure/function [30, 31]. All Ctx+ women were treated with standard doses of chemotherapy agents known to cause CIPN-sx, such as taxanes and platinum compounds. 


\section{Measures}

\section{Demographics and cancer treatment}

Age at baseline (years), education (years), race and ethnicity (categories), and initial stage of breast cancer (0-IIIc) were collected by self-report at baseline. Information on participants' exposure to chemotherapy and other treatments associated with the development of symptoms simlar to CIPN-sx (e.g., muscle/joint pain) were collected from medical records.

\section{Presence, frequency, number, and severity of CIPN-sx}

The presence, frequency, number, and severity of CIPN-sx were measured using the neurotoxicity subscale of the Functional Assessment of Cancer Treatment Gynecological Oncology Group-Neurotoxicity questionnaire (FACT/GOG$\mathrm{Ntx})$ scale, version 4. The Ntx is an 11-item subscale of the FACT/GOG that measures the presence and severity of several common CIPN-sx. Items ask participants to rate the degree of sensory, motor, auditory, and functional CIPN-sx they experienced during the past week on a five-point Likert scale (0 $4)$, corresponding with increasing symptom severity [33, 34]. The reliability and sensitivity of the Ntx subscale for assessing CIPN-sx has been established (Cronbach's $\alpha=0.64-0.86$ ) [35], especially in patients receiving taxanes [34]. Three $\mathrm{Ctx}+$ and four $\mathrm{Ctx}-$ included in the original study were excluded from the analysis because complete data on CIPN-sx or perceived ability to work was not available. Given the unique nature of each question, missing data was not imputed.

The presence of CIPN-sx was determined by calculating the total number of $\mathrm{Ctx}+$ and $\mathrm{Ctx}-$ women who reported having a specific CIPN-sx, regardless of its severity (i.e., any score greater than 0 ). The frequency of CIPN-sx was determined by converting the total number of women who reported having each CIPN-sx at each time point into a percentage. The total number of CIPN-sx at each time point was calculated by tabulating the number of unique CIPN-sx participants reported at each time point, regardless of their severity. The severity of $C I P N$-sx was determined by calculating mean scores for the (a) total FACT/GOG-Ntx scale; (b) total scores for the sensory, motor, hearing, and functional domains; and (c) scores for individual symptoms in each domain at each time point.

\section{Perceived ability to work and employment status}

Participants' perceived ability to work was measured using an item from the Functional Well-Being subscale of the FACT/GOG-Ntx (version 4), which asked participants to respond to the statement "I am able to work (including housework)." Perceived ability to work was scored on a five-point Likert scale $(0=$ not at all, $1=$ a little bit, $2=$ somewhat, $3=$ quite a bit, $4=$ very much)
To help inform the discussion about the impact of CIPN-sx on occupational function, we also evaluated (1) the percentage of women that were working either full-or part-time at each time point, (2) the type of work in which they were engaged (unskilled, semi-skilled, skilled, managerial/clerical/official/sales, or professional/technical), and (3) any change in the type of work performed from baseline to $1 \mathrm{Y}$.

\section{Statistical analyses}

Analyses were performed using SPSS, version 23 (IBM Corporation). Descriptive statistics were used to tabulate the presence (yes/no) and frequency (\%) of CIPN-sx at each time point. Pearson's chi-squared tests were used to compare the frequency of categorical demographic variables and the frequency of CIPN-sx between groups at $1 \mathrm{M}$ and $1 \mathrm{Y}$. For categorical variables with fewer than five observations, Fisher's exact test was substituted. For categorical variables with fewer than five observations where order mattered (i.e., stage of cancer and perceived ability to work), Mantel-Hanzel chi-squared tests were used. Independent sample $t$-tests were used to compare continuous demographic variables, the number of CIPN-sx reported, and total FACT/GOG-Ntx scores. Differences in domain scores and individual items on the FACT/GOG-Ntx were compared using Mann-Whitney $U$ tests, with a Benjamini-Hochberg correction to reduce the risk of type I errors due to multiple comparisons. Differences in ordinal responses to the work item between $\mathrm{Ctx}+$ and $\mathrm{Ctx}^{-}$ were compared using ordinal regression models, using cancer stage as a covariate. Spearman's coefficients $\left(R_{S}\right)$ were used to identify significant correlations between the presence, severity, and number of CIPN-sx and ability to work post-treatment.

Ordinal regression models were constructed to explore the combination of CIPN-sx that best predicted ability to work for $\mathrm{Ctx}+$ at $1 \mathrm{M}$ and $1 \mathrm{Y}$. Because of the small sample size, $1 \mathrm{M}$ and $1 \mathrm{Y}$ models were limited to two CIPN-sx apiece (i.e., one predictor variable per $\sim 10$ observations). Only the severity of CIPN-sx was used in the analyses to minimize the potential to inflate significance levels because of variables with shared sources of variance. Final regression models were selected based on their statistical significance ( $p$ value) and explanatory value (pseudo $r^{2}$ value). Because of the sample size, models were also carefully screened for signs of overfitting (e.g., initial model fit $<0.05$, goodness of fit $>0.05$ ).

\section{Results}

\section{Demographics}

Table 1 summarizes the demographics for the sample. Participants were predominantly middle-aged, white, and well- 
Table 1 Sample characteristics

\begin{tabular}{|c|c|c|c|}
\hline & Ctx + & $\mathrm{Ctx}^{-}$ & Significance \\
\hline Demographics & $N=22$ & $N=22$ & \\
\hline Age at baseline (years, mean (SD)) & $49.68(8.0)$ & $52.68(9.3)$ & 0.256 \\
\hline Race $(\%$, Caucasian) & 77.3 & 86.4 & 0.349 \\
\hline Education (years, mean (SD)) & $15.59(2.8)$ & $15.27(2.4)$ & 0.684 \\
\hline Less than high school $(<12$ years, $\%)$ & 4.5 & 4.5 & - \\
\hline High school (12 years, \%) & 9.1 & 9.1 & - \\
\hline Some college $(13-15$ years, $\%)$ & 36.4 & 27.3 & - \\
\hline Four-year college ( 16 years, $\%)$ & 18.2 & 27.3 & - \\
\hline More than four-year college ( $>16$ years, $\%$ ) & 31.8 & 27.3 & - \\
\hline \multicolumn{4}{|l|}{ Cancer and cancer treatment } \\
\hline \multicolumn{4}{|l|}{ Stage of breast cancer at diagnosis (\%, stages $0-3$ ) } \\
\hline Stage 0 & 0.0 & 18.2 & \\
\hline Stage 1 & 50.0 & 68.2 & $0.002^{\mathrm{c}}$ \\
\hline Stage 2 & 40.9 & 13.6 & \\
\hline Stage 3 & 9.1 & 0.0 & \\
\hline \multicolumn{4}{|l|}{ Chemotherapy } \\
\hline Adjuvant (\%) & 68.2 & 0.0 & - \\
\hline Neo-adjuvant (\%) & 31.8 & 0.0 & - \\
\hline Received chemotherapy (any type, \%) & 100.0 & 0.0 & - \\
\hline Received a single neurotoxic agent during treatment $(\%)$ & 95.5 & 0.0 & - \\
\hline Received two or more neurotoxic agents during treatment (\%) & 31.7 & 0.0 & - \\
\hline \multicolumn{4}{|l|}{ Type of chemotherapy received } \\
\hline Taxane $(\%)$ & 95.5 & 0.0 & - \\
\hline Docetaxel (\%) & 54.5 & 0.0 & - \\
\hline Paclitaxel (\%) & 40.9 & 0.0 & - \\
\hline Platinating agent $(\%)$ & 22.7 & 0.0 & - \\
\hline Carboplatin (\%) & 22.7 & 0.0 & - \\
\hline Received both taxane and platinum $(\%)$ & 22.7 & 0.0 & - \\
\hline Total exposure to neurotoxic chemotherapy $\left(\mathrm{mg} / \mathrm{m}^{2}\right.$, mean (SD)) & $1757.1(1967.3)$ & 0.0 & - \\
\hline Total exposure to a taxane $\left(\mathrm{mg} / \mathrm{m}^{2}\right.$, mean (SD)) & $911.1(491.8)$ & 0.0 & - \\
\hline Amount of paclitaxel $\left(\mathrm{mg} / \mathrm{m}^{2}\right)$ & $452.5(727.6)$ & 0.0 & - \\
\hline Amount of docetaxel $\left(\mathrm{mg} / \mathrm{m}^{2}\right)$ & $388.7(376.9)$ & 0.0 & - \\
\hline Amount of carboplatin $\left(\mathrm{mg} / \mathrm{m}^{2}\right)$ & $958.1(1828.9)$ & 0.0 & \\
\hline \multicolumn{4}{|l|}{ Radiotherapy } \\
\hline Received radiotherapy during treatment $(\%)$ & 81.8 & 59.1 & 0.099 \\
\hline Amount of radiation per fraction (Gy, mean (SD)) & $2.07(0.96)$ & $1.93(0.80)$ & 0.717 \\
\hline \multicolumn{4}{|l|}{ Hormonal therapies } \\
\hline Aromatase inhibitor (AI; \%) & 27.2 & 45.5 & $0.510^{\mathrm{b}}$ \\
\hline Selective estrogen receptor modulators (SERMs; \%) & 22.7 & 36.4 & $0.210^{\mathrm{a}}$ \\
\hline \multicolumn{4}{|l|}{ Biologic agents } \\
\hline Trastuzamab $(\%)$ & 22.7 & 0.0 & $0.048^{\mathrm{b}}$ \\
\hline \multicolumn{4}{|l|}{$\begin{array}{l}\text { Other agents associated with painful and/or CIPN-like } \\
\text { symptoms }\end{array}$} \\
\hline Biphosphanate (\%) & 13.6 & 0.0 & $0.233^{\mathrm{b}}$ \\
\hline
\end{tabular}

$B C$ breast cancer, $S D$ standard deviation, $C t x+$ women with breast cancer treated with surgery and/or radiation and chemotherapy, $C t x$ - women with breast cancer treated with surgery and/or radiation but no chemotherapy

In some cases, totals in a particular category do not equal $100.0 \%$ because participants may have received more than one agent during treatment. Differences between quantitative variables were tested using independent sample $t$-tests, $\alpha=0.05$. Note Values in italics are significant at $\alpha=0.05$

${ }^{a}$ Differences between categorical variables with adequate cell counts were tested with Pearson's chi-squared tests (two-sided), $\alpha=0.05$

${ }^{\mathrm{b}}$ Differences between categorical variables with inadequate cell counts (i.e., $<5$ observations) were tested with Fisher's exact test (two-sided), $\alpha=0.05$

${ }^{\mathrm{c}}$ Differences between categorical variables with inadequate cell counts (i.e., $<5$ observations) where order mattered were tested with Mantel-Hanzel chi-squared tests (two-sided), $\alpha=0.05$

educated. Groups did not differ significantly on age, race, education, use of radiation, amount of radiation received, use of hormonal or biologic therapies, or exposure to other agents commonly given during breast cancer treatment associated with painful or neuropathy-like symptoms (Table 1). Groups differed only on stage of cancer and use of trastuzumab. 
Table 1 also details participant's exposure to cancer treatments associated with CIPN-sx or similar symptoms (e.g., muscle/joint pain). More than $95 \%$ of $\mathrm{Ctx}+$ received at least one neurotoxic agent during treatment, and almost a third received two neurotoxic agents. Of these, all but one received a taxane (docetaxel $54.5 \%$, paclitaxel $40.9 \%$ ). Mean taxane exposure was $911 \pm 492 \mathrm{mg} / \mathrm{m}^{2}$. Five Ctx + who received a taxane also received the platinum compound carboplatin. Mean platinum exposure among Ctx+ was $958.1 \pm 1828.9 \mathrm{mg} / \mathrm{m}^{2}$.

\section{Presence and frequency of CIPN-sx}

At baseline, $\mathrm{Ctx}+$ and $\mathrm{Ctx}-$ did not differ significantly on the presence, frequency, severity, or total number of CIPN-sx (Tables 2 and 3). At $1 \mathrm{M}$, more than $50 \%$ of $\mathrm{Ctx}+$ reported numbness, tingling or discomfort in their hands or feet; joint pain/muscle cramps; weakness; and difficulty feeling the shape of small objects. With the exception of three symptoms (joint pain/muscle cramps, trouble walking, and trouble hearing), $\mathrm{Ctx}+$ reported CIPN more frequently than $\mathrm{Ctx}-$ for all symptoms we evaluated at $1 \mathrm{M}$. At $1 \mathrm{Y}$, the frequency and type of CIPN-sx reported by $\mathrm{Ctx}+$ was very similar to $1 \mathrm{M}$, with more than half of $\mathrm{Ctx}+$ reporting numbness, tingling, or discomfort in their hands or feet; joint pain/muscle cramps; and weakness.

Between $1 \mathrm{M}$ and $1 \mathrm{Y}$, the frequency of some CIPN-sx increased slightly between for $\mathrm{Ctx}+$. At $1 \mathrm{Y}$, a slightly higher percentage of $\mathrm{Ctx}+$ reported hand numbness/tingling (63.6 vs. $59.1 \%$ ), foot numbness/tingling (57.1 vs. $54.5 \%$ ), joint pain/muscle cramps (72.7 vs. $68.2 \%)$, trouble buttoning buttons (33.8 vs. $31.8 \%$ ), and difficulty walking (38.1 vs. $27.3 \%$ ) than at $1 \mathrm{M}$.

\section{Total number of CIPN-sx}

At $1 \mathrm{M}, \mathrm{Ctx}+$ reported an average of $5.59 \pm 3.5 \mathrm{CIPN}-\mathrm{sx}$, compared to $3.14 \pm 2.0 \mathrm{CIPN}_{-\mathrm{sx}}$ for $\mathrm{Ctx}^{-}(p=0.006$; Table 2). A year later, the total number of CIPN-sx Ctx+ reported was virtually unchanged; at $1 \mathrm{Y}, \mathrm{Ctx}+$ reported an average of $5.18 \pm 3.5$ symptoms and $\mathrm{Ctx}-$ reported $3.41 \pm 2.3$ $(p=0.049)$.

Table 2 Frequency and total number of chemotherapy-induced peripheral neuropathy symptoms (CIPN-sx) at baseline, 1-month, and 1-year time points

\begin{tabular}{|c|c|c|c|c|c|c|c|c|c|}
\hline \multirow[t]{2}{*}{ Frequency of CIPN-sx ${ }^{\mathrm{a}}$} & \multicolumn{3}{|c|}{ Baseline (pre-treatment) } & \multicolumn{3}{|c|}{ One-month time point ${ }^{\mathrm{b}}$} & \multicolumn{3}{|c|}{ One-year time point ${ }^{\mathrm{c}}$} \\
\hline & $\begin{array}{l}\mathrm{Ctx}+ \\
\%^{\mathrm{a}}\end{array}$ & $\begin{array}{l}\mathrm{Ctx}^{-} \\
\%^{\mathrm{a}}\end{array}$ & Significance & $\begin{array}{l}\mathrm{Ctx}+ \\
\%^{\mathrm{a}}\end{array}$ & $\begin{array}{l}\mathrm{Ctx}^{-} \\
\%^{\mathrm{a}}\end{array}$ & Significance & $\begin{array}{l}\mathrm{Ctx}+ \\
\%^{\mathrm{a}}\end{array}$ & $\begin{array}{l}\mathrm{Ctx}^{-} \\
\%^{\mathrm{a}}\end{array}$ & Significance \\
\hline \multicolumn{10}{|l|}{ Sensory symptoms } \\
\hline Numbness/tingling in hands & 18.2 & 18.2 & $1.000^{\mathrm{e}}$ & 59.1 & 13.6 & $0.002^{\mathrm{d}}$ & 63.6 & 13.6 & $0.001^{\mathrm{d}}$ \\
\hline Numbness/tingling in feet & 9.1 & 18.2 & $0.664^{\mathrm{e}}$ & 54.5 & 9.1 & $0.001^{\mathrm{d}}$ & 57.1 & 18.2 & $0.008^{\mathrm{d}}$ \\
\hline Discomfort in hands & 13.6 & 22.7 & $0.698^{\mathrm{e}}$ & 54.5 & 18.2 & $0.012^{\mathrm{d}}$ & 52.4 & 27.3 & $0.092^{\mathrm{d}}$ \\
\hline Discomfort in feet & 18.2 & 27.3 & $0.472^{\mathrm{d}}$ & 59.1 & 27.3 & $0.033^{\mathrm{d}}$ & 59.1 & 45.5 & $0.365^{\mathrm{d}}$ \\
\hline \multicolumn{10}{|l|}{ Motor symptoms } \\
\hline Joint pain/muscle cramps & 50.0 & 50.0 & $1.000^{\mathrm{d}}$ & 68.2 & 72.7 & $0.741^{\mathrm{d}}$ & 72.7 & 71.4 & $0.924^{\mathrm{d}}$ \\
\hline Feeling weak all over & 22.7 & 22.7 & $1.000^{\mathrm{d}}$ & 77.3 & 36.4 & $0.006^{\mathrm{d}}$ & 59.1 & 22.7 & $0.014^{\mathrm{d}}$ \\
\hline Trouble walking & 18.2 & 36.4 & $0.176^{\mathrm{d}}$ & 36.4 & 54.5 & $0.226^{\mathrm{d}}$ & 38.1 & 59.1 & $0.169^{\mathrm{d}}$ \\
\hline \multicolumn{10}{|l|}{ Auditory symptoms } \\
\hline Trouble hearing & 18.2 & 40.9 & $0.099^{\mathrm{d}}$ & 27.3 & 45.5 & $0.210^{\mathrm{d}}$ & 27.3 & 54.5 & $0.066^{\mathrm{d}}$ \\
\hline Ringing/buzzing in ears & 9.1 & 0.0 & $0.448^{\mathrm{e}}$ & 36.4 & 4.5 & $0.021^{\mathrm{e}}$ & 33.3 & 4.5 & $0.046^{\mathrm{e}}$ \\
\hline \multicolumn{10}{|l|}{ Functional symptoms } \\
\hline Trouble buttoning buttons & 9.1 & 0.0 & $0.448^{\mathrm{e}}$ & 31.8 & 0.0 & $0.009^{\mathrm{e}}$ & 33.3 & 0.0 & $0.004^{\mathrm{e}}$ \\
\hline Trouble feeling the shape of small objects in hand & 9.1 & 27.7 & $0.412^{\mathrm{e}}$ & 54.5 & 31.8 & $0.128^{\mathrm{d}}$ & 31.8 & 27.3 & $0.741^{\mathrm{d}}$ \\
\hline \multicolumn{10}{|l|}{ Total number of CIPN-sx } \\
\hline Mean (SD) & $1.95(2.4)$ & 2.59 & $0.366^{\mathrm{f}}$ & $5.59(3.5)$ & 3.14 & $0.006^{\mathrm{f}}$ & $5.18(3.5)$ & 3.41 & $0.049^{\mathrm{f}}$ \\
\hline
\end{tabular}

Note Values in italics are significant at $\alpha=0.05$

$C t x+$ women with breast cancer treated with surgery and/or radiation and chemotherapy, $C t x-$ women with breast cancer treated with surgery and/or radiation but no chemotherapy, $S D$ standard deviation

${ }^{a}$ Frequency is defined as the percentage of $\mathrm{Ctx}+$ or $\mathrm{Ctx}-$ reporting this CIPN-sx in the last 7 days of this time point, regardless of severity

${ }^{\mathrm{b}}$ One month following chemotherapy completion or yoked interval for $\mathrm{Ctx}^{-}$

${ }^{\mathrm{c}}$ One year following 1-month time point

${ }^{\mathrm{d}}$ Differences between percentage of $\mathrm{Ctx}+$ and $\mathrm{Ctx}-$ reporting individual CIPN-sx with adequate cell counts were tested using Pearson's chi-squared tests, $\alpha=0.05$

${ }^{\mathrm{e}}$ Differences between nominal variables with inadequate cell counts (i.e., $<5$ observations) were tested with Fisher's exact test (two-sided), $\alpha=0.05$

${ }^{\mathrm{f}}$ Differences in the total CIPN-sx reported by $\mathrm{Ctx}+$ and $\mathrm{Ctx}-$ at each time point were tested using independent measured $t$ test $(\alpha=0.05)$ 


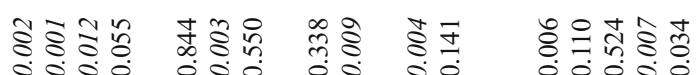

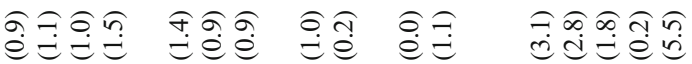

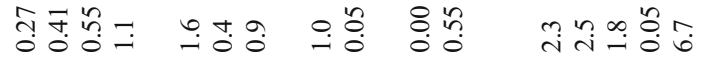

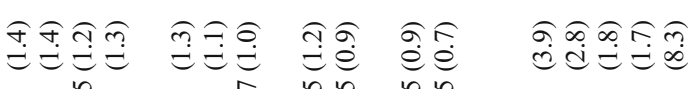

m J哭

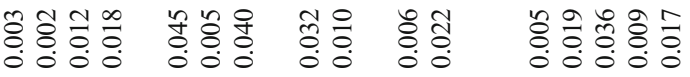

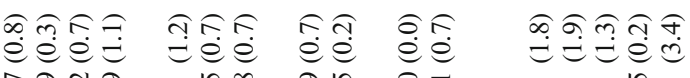

तิ

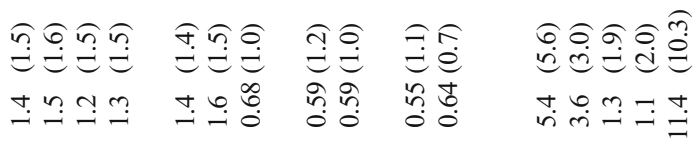

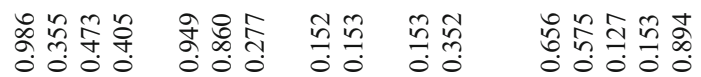

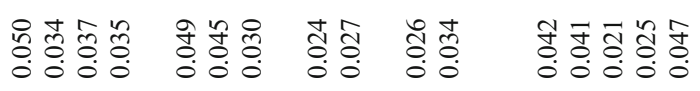

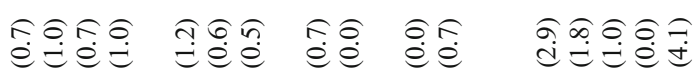

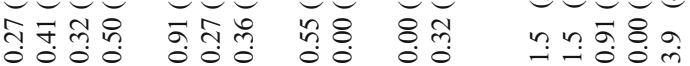

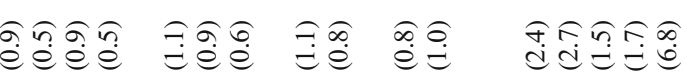

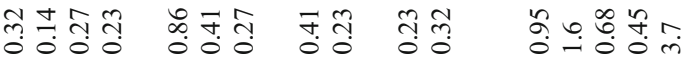
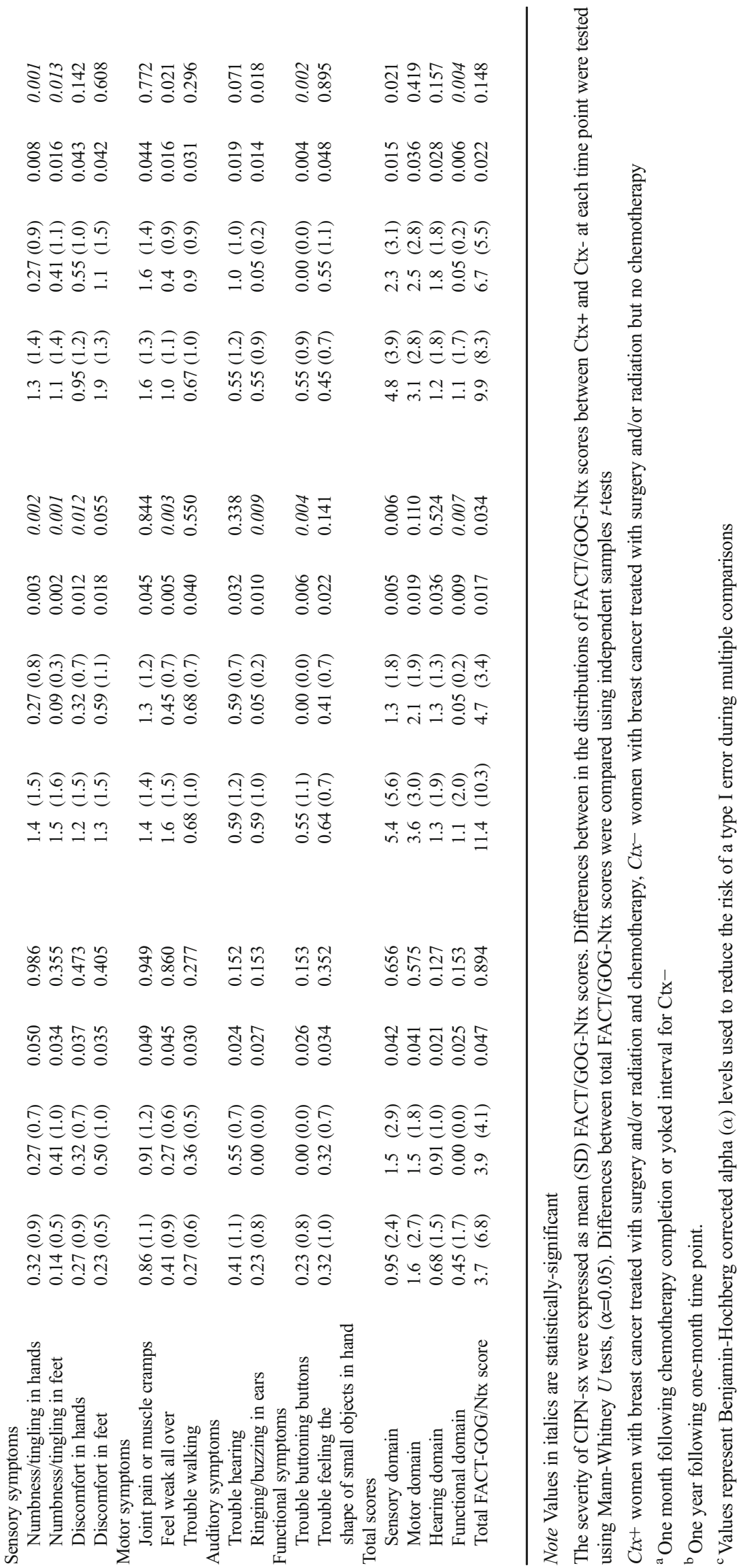


\section{Severity of CIPN-sx}

At $1 \mathrm{M}, \mathrm{Ctx}+$ reported significantly more severe functional CIPN-sx than $\mathrm{Ctx}^{-}(p=0.007)$. In addition, total scores on the sensory domain just missed the cutoff for statistical significance after adjusting for multiple comparisons $(p=0.006$; Table 3). Inspection of individual FACT/GOG-Ntx scores revealed that $\mathrm{Ctx}+$ had more severe numbness/tingling in their hands $(p=0.002)$ and feet $(p=0.001)$, hand discomfort $(p=0.012)$, weakness $(p=0.003)$, ringing/buzzing in their ears $(p=0.009)$, and trouble buttoning buttons $(p=0.004)$ than $\mathrm{Ctx}$ - after treatment.

At $1 \mathrm{Y}$, while total and sensory FACT-GOG/Ntx scores were not significantly different between $\mathrm{Ctx}+$ and $\mathrm{Ctx}-$, $\mathrm{Ctx}+$ continued to report more severe functional CIPN-sx than $\mathrm{Ctx}-(p=0.004)$, including more severe numbness/tingling in their hands $(p=0.001)$ and feet $(p=0.013)$ and trouble buttoning buttons $(p=0.002$; Table 3$)$.

\section{Employment status and perceived ability to work}

At baseline, $1 \mathrm{M}$, and $1 \mathrm{Y}$, all $44 \mathrm{Ctx}+$ and $\mathrm{Ctx}$ - were working part-or full-time (Fig. 1). Of these, $92.4 \%$ of $\mathrm{Ctx}+$ and more than $70 \%$ of $\mathrm{Ctx}^{-}$were working in positions classified as either professional/technical or managerial/official/clerical/ sales in nature (Fig. 2). Over the course of the study (i.e., BL to $1 \mathrm{Y}$ ), there was virtually no change in the type of work participants performed (data not shown).

Compared to survivors who reported being "very" able to work at $1 \mathrm{M}$, having received chemotherapy during treatment significantly predicted being only "somewhat" $(p=0.00)$, "a little bit" $(p=0.00)$, or "not at all" ( $p=0.03)$ able to work, after controlling for differences in cancer stage between $\mathrm{Ctx}+$ and $\mathrm{Ctx}^{-}$(data not shown). Similarly, at $1 \mathrm{Y}$ compared to women who were very able to work, having received chemotherapy during treatment was also significantly associated with being only "a little" or somewhat able to work, after controlling for stage of cancer. This difference was especially evident at $1 \mathrm{M}$, where $50 \%$ of $\mathrm{Ctx}+$ reported being only somewhat, a little bit, or not at all able to work, compared to just $9.1 \%$ of $\mathrm{Ctx}^{-}$survivors.

\section{Association between presence, severity, and number of CIPN-sx and ability to work}

At $1 \mathrm{M}$, the severity of the following five combinations of CIPN-sx were correlated with BCS' work ability: hand numbness/tingling $\left(R_{S}=-0.483 ; p=0.023\right)$, hand discomfort $\left(R_{S}=-0.511 ; p=0.015\right)$, weakness $\left(R_{S}=-0.557\right.$; $p=0.007)$, trouble hearing $\left(R_{s}=-0.454 ; p=0.034\right)$, and difficulty feeling the shape of small objects $\left(R_{s}=-0.463\right.$; $p=0.030$ ). In addition, at $1 \mathrm{M}$ the presence of any hand discomfort $\left(R_{s}=-0.455 ; p=0.033\right)$, weakness $\left(R_{S}=-0.603 ; p=0.003\right)$, trouble hearing $\left(R_{S}=-0.501\right.$; $p=0.018)$, or trouble feeling the shape of small objects in hand $\left(R_{s}=-0.433 ; p=0.044\right)$, regardless of severity, were also significantly correlated with work scores among $\mathrm{Ctx}+$, as was the number of CIPN-sx reported at $1 \mathrm{M}$ $\left(R_{s}=-0.526 ; p=0.012\right)$.

At $1 \mathrm{Y}$, only the presence of weakness (regardless of severity) was associated with perceived work ability for $\mathrm{Ctx}+$ $\left(R_{s}=-0.478 ; p=0.024\right)$.

\section{Using the severity of CIPN-sx to predict work ability post-treatment}

Results of the exploratory analysis using ordinal regression identified five models that predicted $\mathrm{Ctx}+$ who were not at all able to work at $1 \mathrm{M}$, which are presented in Table 4: (1) hand numbness and trouble feeling the shape of small objects (Wald $\chi^{2}(1)=11.39$; cumulative $\mathrm{OR}=0.008$; Nagelkerke $\left.r^{2}=0.500\right)$, (2) trouble buttoning buttons and trouble feeling the shape of small objects (Wald $\chi^{2}(1)=12.99 ;$ OR $=0.004$; Nagelkerke $\left.r^{2}=0.567\right),(3)$ foot numbness and foot pain (Wald $\chi^{2}(1)=7.65$;
Fig. 1 Employment status, type of work, and perceived ability to work at baseline, 1-month, and 1year time points. The figure illustrates the percentages of $\mathrm{Ctx}+$ and $\mathrm{Ctx}^{-}$, respectively, who were classified as working in each type of work (unskilled, semi-skilled, skilled, managerial/clerical/ official, or professional/technical) at each time point (BL, $1 \mathrm{M}, 1 \mathrm{Y}$ )

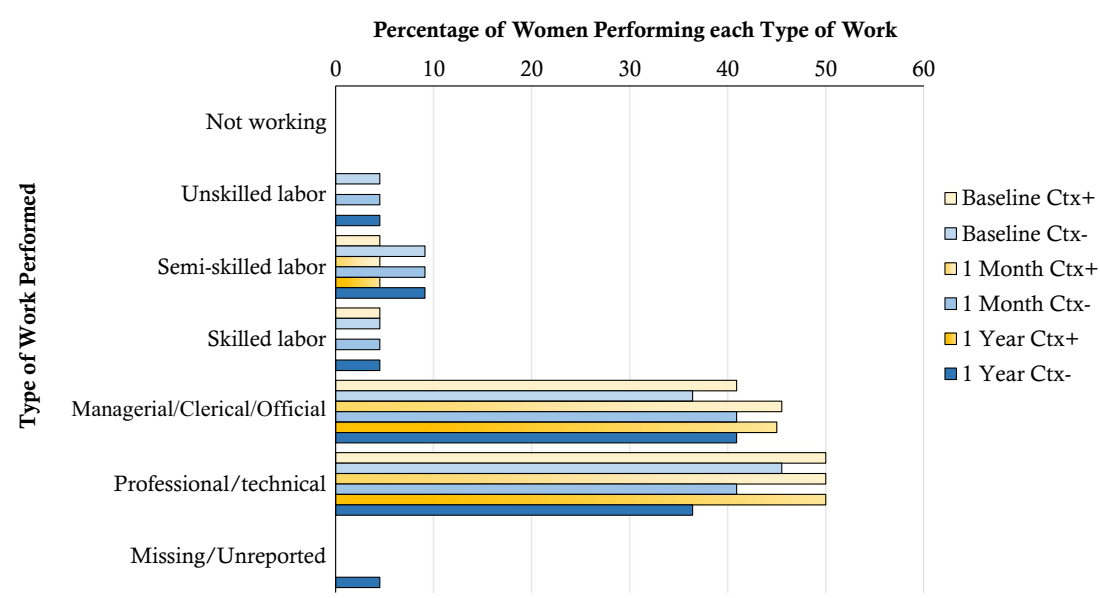


Fig. 2 Perceived ability to work at baseline, 1-month, and 1-year time points. The figure illustrates the percentage of $\mathrm{Ctx}+$ and $\mathrm{Ctx}^{-}$ who reported being not at all, a little bit, somewhat, quite a bit, and very much able to work (including housework) at each time point

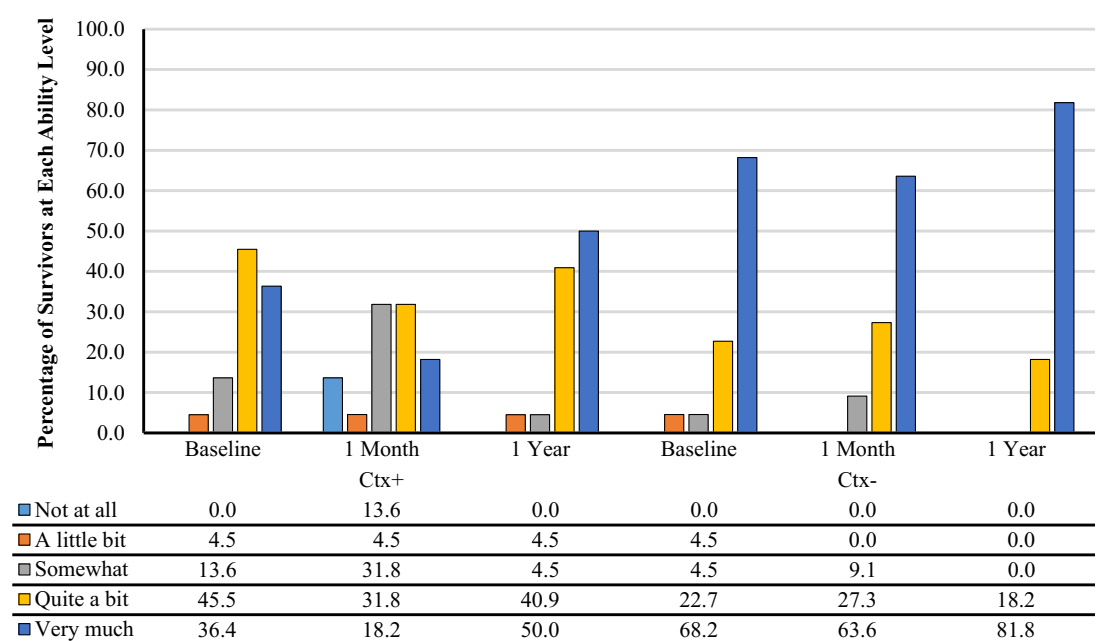

OR $=0.031$; Nagelkerke $\left.r^{2}=0.644\right)$, (4) foot numbness and trouble walking (Wald $\chi^{2}(1)=10.67$; OR $=0.003$; Nagelkerke $\left.r^{2}=0.724\right)$, and (5) trouble hearing and hand pain (Wald $\chi^{2}(1)=12.02 ;$ OR $=0.005$; Nagelkerke $\left.r^{2}=0.583\right)$. At $1 \mathrm{Y}$, no combination of CIPN-sx significantly predicted work ability for $\mathrm{Ctx}+$ (data not shown).

Table 4 Ordinal regression models using the severity of CIPN-sx to predict perceived work ability in chemotherapy-treated (Ctx+) breast cancer survivors approximately 1 month after treatment $(N=22)$

\begin{tabular}{|c|c|c|c|c|c|c|c|c|}
\hline \multirow[t]{2}{*}{ Variable (reference) } & \multirow[t]{2}{*}{ Coefficient } & \multirow[t]{2}{*}{ SE } & \multirow[t]{2}{*}{ Wald } & \multirow[t]{2}{*}{$p$ value } & \multicolumn{2}{|c|}{$95 \% \mathrm{CI}$} & \multirow{2}{*}{$\begin{array}{l}\text { Cumulative } \\
\text { odds ratio }\end{array}$} & \multirow[t]{2}{*}{ Nagelkerke $r^{2}$} \\
\hline & & & & & Lower & Upper & & \\
\hline \multicolumn{9}{|l|}{ Combination 1 , hand symptoms } \\
\hline Ability to work (not at all) & -4.24 & 1.26 & 11.39 & 0.001 & -6.70 & -1.78 & 0.008 & 0.500 \\
\hline Hand numbness (somewhat) & -4.50 & 1.89 & 5.64 & 0.018 & -8.21 & -.786 & & \\
\hline Trouble feeling the shape of small objects (very much) & 7.28 & 3.13 & 5.43 & 0.020 & 1.16 & 13.41 & & \\
\hline \multicolumn{9}{|l|}{ Combination 2, hand symptoms } \\
\hline Ability to work (not at all) & -5.50 & 1.53 & 12.99 & 0.000 & -8.50 & -2.51 & 0.004 & 0.567 \\
\hline Trouble buttoning buttons (a little bit) & -3.79 & 1.60 & 5.60 & 0.018 & -6.93 & -0.65 & & \\
\hline Trouble feeling the shape of small objects (somewhat) & -4.48 & 1.87 & 5.75 & 0.017 & -8.15 & -0.82 & & \\
\hline \multicolumn{9}{|l|}{ Combination 3 , lower extremity } \\
\hline Ability to work (not at all) & -3.47 & 1.26 & 7.65 & 0.006 & -5.94 & -1.01 & 0.031 & 0.644 \\
\hline Foot numbness (somewhat) & -5.98 & 2.36 & 6.40 & 0.011 & -10.6 & -1.35 & & \\
\hline Foot pain (quite a bit) & 9.36 & 4.21 & 4.95 & 0.026 & 1.12 & 17.60 & & \\
\hline \multicolumn{9}{|l|}{ Combination 4 , lower extremity } \\
\hline Ability to work (not at all) & -5.79 & 1.77 & 10.67 & 0.001 & -9.26 & -2.32 & 0.003 & 0.724 \\
\hline Foot numbness (quite a bit) & -4.68 & 1.83 & 6.52 & 0.011 & -8.28 & -1.09 & & \\
\hline Trouble walking (quite a bit) & 4.40 & 2.12 & 4.33 & 0.037 & 0.26 & 8.55 & & \\
\hline \multicolumn{9}{|l|}{ Combination 5 , hearing and hand pain } \\
\hline Ability to work (not at all) & -5.32 & 1.54 & 12.02 & 0.001 & -8.33 & -2.31 & 0.005 & 0.583 \\
\hline Hand pain (very much) & -3.94 & 1.72 & 5.26 & 0.022 & -7.32 & -0.57 & & \\
\hline Trouble hearing (a little bit) & -4.31 & 1.85 & 5.45 & 0.020 & -7.94 & -0.69 & & \\
\hline
\end{tabular}

Values represent the results of separate univariate ordinal regression models using the severity of individual CIPN-sx as predictor variables and selfreported ability to work (including housework) as the dependent variable (categories: not at all, a little bit, somewhat, quite a bit, very much). To ensure that models predicted poor work performance based on the severity CIPN-sx, CIPN-sx were reverse coded (i.e., $0=$ very much, $1=$ quite a bit, $2=$ somewhat, $3=$ a little bit, $4=$ not at all). Note Values in italics are significant at $\alpha=0.05$

$S E$ standard error, $C I$ confidence interval 


\section{Discussion}

There is growing evidence that CIPN-sx pose a risk to BCS not only during cancer treatment but after as well [24-26, 36-41]. Previous research has linked CIPNsx with poorer quality of life and decreased ability to function [28], but this is one of the first studies to address the question of whether CIPN-sx impact work in BCS post-treatment [28]. Results of this study add to the growing evidence showing the persistence of CIPN-sx after treatment $[25,26,36]$, and extend these findings to the context of occupational function, demonstrating a clear link between the presence, frequency, number, and severity of CIPN-sx and BCS' self-reported inability to work following treatment. Results of our analysis also suggest that (1) the total number of CIPN-sx Ctx+ report, regardless of their severity, may be a useful predictor of difficulty working and that (2) painful and non-painful CIPNsx affecting the hands or feet (with or without treatmentrelated hearing deficits) may predict work difficulty for $\mathrm{Ctx}+1 \mathrm{M}$ post-treatment.

Presence and frequency of CIPN-sx An important finding from our analysis was that the pattern of CIPN-sx reported by $\mathrm{Ctx}+$ approximately a year post-treatment was very similar to the pattern reported at $1 \mathrm{M}$. In the context of work, this has important implications because it raises the possibility that the CIPN-sx survivors present with immediately after treatment may be a reliable indicator of the CIPN-sx that will continue to interfere with a survivor's ability to work during the critical first year of survivorship. Research in larger samples is needed to test this hypothesis.

Total number of CIPN-sx A second potentially important finding from our analysis was that the number of CIPN-sx $\mathrm{Ctx}+$ reported, independent of their severity, appeared to predict ability to work post-treatment. The majority of studies have used either the presence $[14,15,29]$ or severity of CIPN-sx as the primary lens for evaluating CIPN-related outcomes [41]. While our results suggest that both are likely to predict work ability, the number of CIPN-sx patient's experience itself may also be disruptive to work. On average, $\mathrm{Ctx}+$ in our sample reported five different CIPN-sx at both posttreatment time points. As with other studies, when we looked at the severity of these CIPN-sx, in many cases, we observed a pattern of one or two severe CIPN-sx and several milder symptoms [41]. Because this mixed severity pattern is common, it will be important to clarify whether the number of CIPN-sx survivors report is just as predictive of difficulty working as severity of each symptom. This is not only because the severity of CIPN-sx can vary but also because CIPN-sx often grow less severe over time [10], which could lead providers to overlook the potentially serious impact that a number of milder CIPN-sx could have on BCS' ability to work.

Association between the severity of CIPN-sx and ability to work at $1 \mathrm{M}$ During our regression analyses, we identified several combinations of CIPN-sx that were predictive of $\mathrm{Ctx}+$ that were less able to work at $1 \mathrm{M}$. These included two combinations of sensory CIPN-sx affecting the hands, two combinations of CIPN-sx affecting the feet, and the combination of hearing loss and hand discomfort (Table 4). These findings are consistent with the few studies of CIPN in which work was considered [28] and make sense intuitively. In particular, the finding that sensory symptoms that interfere with women's ability to feel the shape of objects or button buttons were associated with difficulty working was not surprising. These combinations of CIPN-sx point to potential phenotypes for CIPN-related work interference. It is important to note that several other symptoms such as weakness and joint pain/muscle soreness also predicted inability to work at $1 \mathrm{M}$ in several of our models but were not included because of lack of model fit given our sample size. Statistical considerations notwithstanding, it is clear that symptoms such as joint pain/muscle soreness and weakness have the potential to impact work and should be included in future studies of CIPN-sx and work.

Association between the severity of CIPN-sx and ability to work at $1 \mathrm{Y}$ There are several reasons that may explain why we did not observe a stronger effect of CIPN-sx on perceived work ability at $1 \mathrm{Y}$. First, the percentage of $\mathrm{Ctx}+$ who reported being "very much" or "quite a bit" able to work after treatment rose from $41.4 \%$ at $1 \mathrm{M}$ to $90.9 \%$ at $1 \mathrm{Y}$ (Fig. 2a). At the same time, the type, frequency, severity, and number of CIPN-sx Ctx+ reported remained relatively constant. This combination of improving ability to work in the face of relatively stable CIPN-sx suggests that while BCS may continue to experience CIPN post-treatment, the impact of these symptoms on work may lessen over time as BCS acclimate to their symptoms and/or develop coping strategies.

Finally, it is important to note that while the lack of treatments for CIPN-sx is problematic on many fronts, it is particularly concerning in the context of work. Recently, several groups have had some success treating the painful component of CIPN-sx non-invasively using devices such as scrambler therapy [42], as well as several oral or topical agents [10]. These successes raise the question of whether strategies like these could be used to address painful CIPN-sx before they can disrupt cancer survivors' transition back to usual work activities. 


\section{Limitations}

While this study provides a useful starting point for future research exploring the impact of CIPN-sx on breast cancer survivor's ability to work post-treatment, our results need to be considered in light of several limitations. First, the sample for this analysis was small; larger samples will be needed to validate these findings and explore which combinations of CIPN-sx best predict survivor's ability to work. Secondly, we did not have access to detailed information on potential risk factors for CIPN such as osteoarthritis, and the size of our sample prevented us from including symptoms such as depression as covariates in our analysis, both of which should be included in future studies. Third, our analysis relied on a single item to evaluate perceived ability to work post-treatment. While this item provided a useful lens for looking at the impact of CIPN-sx on work, clearly, more stringent and varied measures of work including absenteeism, productivity, and performance on jobspecific tasks will be needed to understand the occupational impact of CIPN-sx. In addition, we did not have access to objective measures of CIPN, which would have provided valuable insight into structural or functional changes in nerves that may help to explain perceived difficulties working. Fourth, our sample was racially/ ethnically homogeneous; more diverse cohorts will be needed to understand whether the impact of CIPN-sx on work outcomes differs by race, ethnicity, or culture. Finally, our sample was approximately 10 years younger than the median age of BCS in the USA. The reason for this was that the study upon which our analysis was based enrolled only women younger than age 70. As such, while our sample is representative of working-age BCS, studies in older survivors that continue work past the current typical age of retirement will be needed to explore the impact of CIPN-sx on work in this population fully.

\section{Conclusion}

The increasing reliance on neurotoxic chemotherapy to treat many forms of breast cancer means that clinicians and patients will need to carefully consider how best to balance the benefits and risks of treatment, including the potential impact of CIPNsx on their ability to work. Our findings suggest that clinicians should remain vigilant for CIPN-sx that may interfere with women's ability to work during the first year post-treatment, when many survivors return to work. Women who continue to experience CIPN-sx post-treatment (especially in the context of other symptoms that can interfere with work such as pain, fatigue, or cognitive disruption) may need to be referred to physical or occupational therapy to minimize the negative impact of CIPN-sx on work.

This concern may be especially pertinent in the modern workplace, with its growing reliance on tactile technologies such as keyboards and touch screens, which require users to be able to effortlessly tap, touch, type, and glide their way through their workday. Identifying the specific CIPN-sx and dimension of the symptom experience (i.e., presence, frequency, severity, or total number) that best predict difficulty working is an essential first step towards developing interventions to reduce their impact on work for BCS with CIPN.

Acknowledgments This study was partially supported by the National Cancer Institute (R01 CA101318, R01 CA087845, P30 CA082709, and R25 CA117865) and the Indiana CTSI (UL1 RR025761, S10 RR027710, and C06 RR020128) with additional support from the National Institute on Aging (R01 AG019771, P30 AG010133). The authors thank the Indiana University Melvin and Bren Simon Cancer Center recruitment core and our oncologist colleagues for their invaluable assistance with patient recruitment. Finally, we are grateful to our participants for their time and effort; this research would not have been possible without their willingness to participate during a particularly challenging time in their lives.

\section{Compliance with ethical standards}

Conflict of interest The authors have no conflicts of interest to disclose. The authors verify that they have full control of all primary data and agree, if requested, to allow the journal to verify the data from which all analyses were derived.

Open Access This article is distributed under the terms of the Creative Commons Attribution-NonCommercial 4.0 International License (http:// creativecommons.org/licenses/by-nc/4.0/), which permits any noncommercial use, distribution, and reproduction in any medium, provided you give appropriate credit to the original author(s) and the source, provide a link to the Creative Commons license, and indicate if changes were made.

\section{References}

1. Islam T et al. (2014) Factors associated with return to work of breast cancer survivors: a systematic review. BMC Public Health 14(Suppl 3):S8

2. Spelten ER, Sprangers MA, Verbeek JH (2002) Factors reported to influence the return to work of cancer survivors: a literature review. Psychooncology 11(2):124-131

3. Tamminga SJ et al. (2012) Breast cancer survivors' views of factors that influence the return-to-work process - a qualitative study. Scand J Work Environ Health 38(2):144-154

4. Ahn E et al. (2009) Impact of breast cancer diagnosis and treatment on work-related life and factors affecting them. Breast Cancer Res Treat 116(3):609-616

5. Banning M (2011) Employment and breast cancer: a meta-ethnography. Eur J Cancer Care (Engl) 20(6):708-719

6. Bradley CJ et al. (2005) Short-term effects of breast cancer on labor market attachment: results from a longitudinal study. J Health Econ 24(1):137-160 
7. Mahon, S.M., et al. (2011) Breast cancer, in Site specific series, S.M. Mohan, Editor. Oncology Nursing Society. 248

8. de Boer, A.G., et al. (2011) Interventions to enhance return-to-work for cancer patients. Cochrane Database Syst Rev (2): p. CD007569

9. Wolf SL et al. (2012) The relationship between numbness, tingling, and shooting/burning pain in patients with chemotherapy-induced peripheral neuropathy (CIPN) as measured by the EORTC QLQCIPN20 instrument, N06CA. Support Care Cancer 20(3):625-632

10. Hershman DL et al. (2014) Prevention and management of chemotherapy-induced peripheral neuropathy in survivors of adult cancers: American Society of Clinical Oncology clinical practice guideline. J Clin Oncol 32(18):1941-1967

11. Mols, F., et al. (2014) Chemotherapy-induced peripheral neuropathy and its association with quality of life: a systematic review. Support Care Cancer

12. Driessen CM et al. (2012) Assessing the impact of chemotherapyinduced peripheral neurotoxicity on the quality of life of cancer patients: the introduction of a new measure. Support Care Cancer 20(4):877-881

13. Sorbe B et al. (2012) A phase II study of docetaxel weekly in combination with carboplatin every three weeks as first line chemotherapy in stage IIB-IV epithelial ovarian cancer: neurological toxicity and quality-of-life evaluation. Int J Oncol 40(3):773-781

14. Bhatnagar B et al. (2014) Chemotherapy dose reduction due to chemotherapy induced peripheral neuropathy in breast cancer patients receiving chemotherapy in the neoadjuvant or adjuvant settings: a single-center experience. Springerplus 3:366

15. Speck RM et al. (2013) Impact of chemotherapy-induced peripheral neuropathy on treatment delivery in nonmetastatic breast cancer. $\mathrm{J}$ Oncol Pract 9(5):e234-e240

16. Dougherty PM et al. (2004) Taxol-induced sensory disturbance is characterized by preferential impairment of myelinated fiber function in cancer patients. Pain 109(1-2):132-142

17. Tofthagen $\mathrm{C}$ (2010) Patient perceptions associated with chemotherapy-induced peripheral neuropathy. Clin J Oncol Nurs 14(3):E22-E28

18. United States Department of Labor ( 2016) Labor force by sex, 2014 and projected 2022. [cited 2016 April 25]; Available from: http://www.dol.gov/wb/stats/laborforce sex projected_2014_2022_txt.htm

19. American Cancer Society (2016) Facts and figures, A.C. Society, Editor. Atlanta

20. Bradley CJ et al. (2007) Employment and cancer: findings from a longitudinal study of breast and prostate cancer survivors. Cancer Investig 25(1):47-54

21. Chirikos TN, Russell-Jacobs A, Jacobsen PB (2002) Functional impairment and the economic consequences of female breast cancer. Women Health 36(1):1-20

22. Forsyth PA et al. (1997) Prospective study of paclitaxel-induced peripheral neuropathy with quantitative sensory testing. J NeuroOncol 35(1):47-53

23. Park, S.B., et al. (2013) Chemotherapy-induced peripheral neurotoxicity: a critical analysis. CA Cancer J Clin

24. Osmani K et al. (2012) Taxane-induced peripheral neuropathy has good long-term prognosis: a 1- to 13-year evaluation. J Neurol 259(9):1936-1943

25. Pignata $\mathrm{S}$ et al. (2006) Residual neurotoxicity in ovarian cancer patients in clinical remission after first-line chemotherapy with carboplatin and paclitaxel: the Multicenter Italian Trial in ovarian cancer (MITO-4) retrospective study. BMC Cancer 6:5
26. Hershman DL et al. (2011) Association between patient reported outcomes and quantitative sensory tests for measuring long-term neurotoxicity in breast cancer survivors treated with adjuvant paclitaxel chemotherapy. Breast Cancer Res Treat 125(3):767-774

27. Smith, E.M., et al. (2013) Effect of duloxetine on pain, function, and quality of life among patients with chemotherapy-induced painful peripheral neuropathy: a randomized clinical trial, in JAMA. United States. 1359-67

28. Tanay, M.A., J. Armes, and E. Ream (2016) The experience of chemotherapy-induced peripheral neuropathy in adult cancer patients: a qualitative thematic synthesis. Eur J Cancer Care (Engl)

29. Pike CT et al. (2012) Healthcare costs and workloss burden of patients with chemotherapy-associated peripheral neuropathy in breast, ovarian, head and neck, and nonsmall cell lung cancer. Chemother Res Pract 2012:913848

30. Nudelman KN et al. (2014) Altered cerebral blood flow one month after systemic chemotherapy for breast cancer: a prospective study using pulsed arterial spin labeling MRI perfusion. PLoS One 9(5): e96713

31. Nudelman KN et al. (2016) Cerebral perfusion and gray matter changes associated with chemotherapy-induced peripheral neuropathy. J Clin Oncol 34(7):677-683

32. McDonald BC et al. (2013) Frontal gray matter reduction after breast cancer chemotherapy and association with executive symptoms: a replication and extension study. Brain Behav Immun 30(Suppl):S117-S125

33. Smith, E.M. (2013) Current methods for the assessment and management of taxane-related neuropathy, in Clin J Oncol Nurs. United States. $22-34$

34. Huang HQ et al. (2007) Validation and reduction of FACT/GOGNtx subscale for platinum/paclitaxel-induced neurologic symptoms: a gynecologic oncology group study. Int J Gynecol Cancer 17(2):387-393

35. Griffith KA et al. (2010) Measures of chemotherapy-induced peripheral neuropathy: a systematic review of psychometric properties. J Peripher Nerv Syst 15(4):314-325

36. Beijers A et al. (2014) Chemotherapy-induced peripheral neuropathy and impact on quality of life 6 months after treatment with chemotherapy. J Community Support Oncol 12(11):401-406

37. Park SB et al. (2011) Long-term neuropathy after oxaliplatin treatment: challenging the dictum of reversibility. Oncologist 16(5): 708-716

38. Calhoun EA et al. (2003) Psychometric evaluation of the Functional Assessment of Cancer Therapy/Gynecologic Oncology GroupNeurotoxicity (FACT/GOG-Ntx) questionnaire for patients receiving systemic chemotherapy. Int J Gynecol Cancer 13(6):741-748

39. Kuroi K et al. (2009) Prospective assessment of chemotherapyinduced peripheral neuropathy due to weekly paclitaxel in patients with advanced or metastatic breast cancer (CSP-HOR 02 study). Support Care Cancer 17(8):1071-1080

40. Argyriou AA et al. (2005) Paclitaxel plus carboplatin-induced peripheral neuropathy. A prospective clinical and electrophysiological study in patients suffering from solid malignancies. J Neurol 252(12):1459-1464

41. Bakitas, M.A. (2007) Background noise: the experience of chemotherapy-induced peripheral neuropathy, in Nurs Res. United States. 323-31

42. Marineo G et al. (2012) Scrambler therapy may relieve chronic neuropathic pain more effectively than guideline-based drug management: results of a pilot, randomized, controlled trial. J Pain Symptom Manag 43(1):87-95 